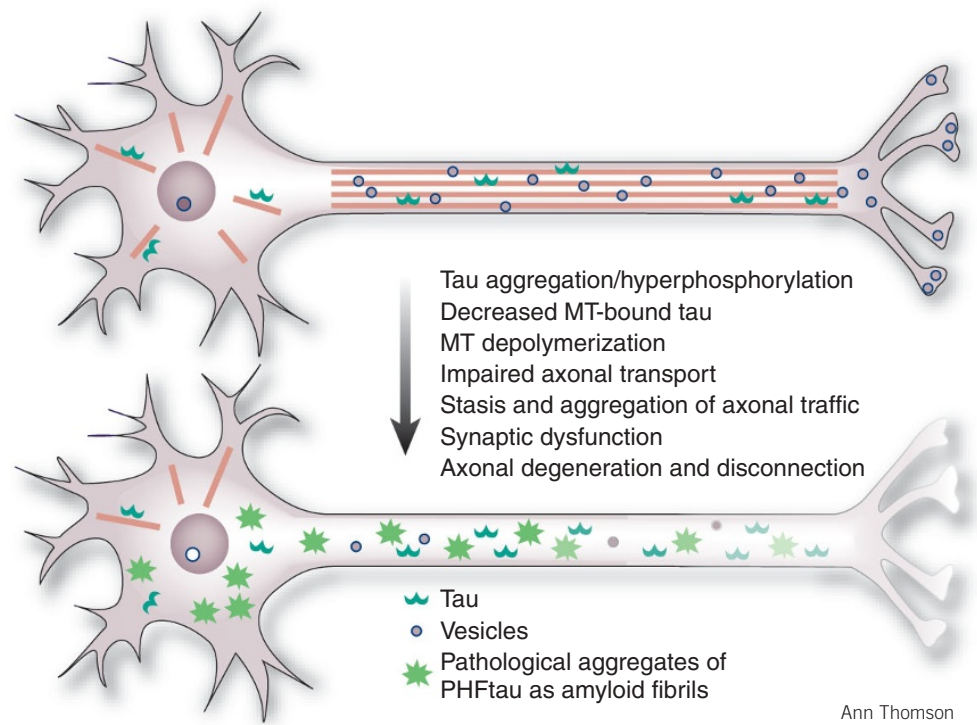

Figure 1 A schematic of the misfolding, fibrillization and sequestration of tau into neurofibrillary tangles and neuritic tau pathology. Top, a normal neuron, in which normal tau maintains the network of microtubules (MTs) that support intraneuronal transport. Bottom, a diseased neuron, in which misfolded tau has formed amyloid fibrils that deposit as neurofibrillary tangles and neuritic tau pathology. The deleterious effects of tau pathology could result from toxic gains of functions by neurofibrillary tangles and neuritic tau pathology or from a loss of tau functions.

possibility that pathological tau may induce neurodegeneration owing to loss-of-function defects by PHFtau rather than as a result of toxic gains of functions by accumulating neurofibrillary tangles.

Significantly, these and other insights into mechanisms of neurodegeneration in Alzheimer disease and related tauopathies suggest that tau pathologies may be tractable or 'druggable' targets for the discovery of new therapies for these conditions ${ }^{4,6}$. For example, microtubule-stabilizing drugs could provide therapeutic benefits in these disor- ders by offsetting the loss of tau function by causing its sequestration in neurofibrillary tangles and dystrophic neurites, or its excessive phosphorylation ${ }^{11}$, whereas microtubule-stabilizing drugs such as paclitaxel also may ameliorate the neurotoxic effects of $\mathrm{A} \beta$ in Alzheimer disease $\mathrm{e}^{12}$. Moreover, additional strategies to develop therapies that target tau abnormalities are being investigated, including high-throughput screening of large compound libraries to identify drugs that not only block tau fibrillization and aggregation but also reduce tau abundance or tau phos- phorylation $^{13-15}$. Notably, like microtubulestabilizing drugs, inhibitors of tau kinases, such as glycogen synthase kinase-3, may be able to block pathways that lead to the formation of both $A \beta$ and tau amyloid lesions in Alzheimer disease ${ }^{4}$.

Perhaps most importantly, the work of SantaCruz et al. suggests that rectifying losses of tau function may be as valuable as, or more valuable than, ameliorating gains of toxic properties by neurofibrillary tangles in drug-discovery programs for Alzheimer disease and related tauopathies. Indeed, if this lesson can be extrapolated to other neurodegenerative brain amyloidoses, researchers may now need to pay more attention to the normal functions of disease proteins such as tau, $\mathrm{A} \beta, \alpha$-synuclein, prions, and so on to fully understand the role of pathologically altered forms of these proteins in mechanisms of neurodegeneration.

1. Dobson, C.M. Nature 426, 884-890 (2003).

2. Forman, M.S., Trojanowski, J.Q. \& Lee, V.M.-Y. Nat Med. 10, 1055-1063 (2004).

3. Selkoe, D.J. Nat. Cell Biol. 6, 1054-1061 (2004).

4. Skovronsky, D.M., Lee, V.M.-Y. \& Trojanowski, J.Q. Annu. Rev. Pathol. (in the press).

5. SantaCruz, K. et al. Science 309, 476-481 (2005).

6. Fillit, H.M. \& Refolo, L.M. Curr. Alzheimer Res. 2, 105-109 (2005).

7. Lansbury, P.T. Jr. Nat. Med. 10 (Suppl.), S51-S57 (2004).

8. Xiong, G. \& Doraiswamy, P.M. Geriatrics $60,22-26$ (2005).

9. Lee, V.M.-Y. \& Trojanowski, J.Q. Curr. Opin. Neurobiol. 2, 653-656 (1992)

10. Mitchell, T.W. et al. J. Histochem. Cytochem. 48 1627-1638 (2000)

11. Zhang, B. et al. Proc. Natl. Acad. Sci. USA 102 227-231 (2005).

12. Michaelis, M.L. et al. J. Pharmacol. Exp. Ther. 312 659-668 (2005).

13. Dickey, C.A. et al. Curr. Alzheimer Res. 2, 231-239 (2005).

14. Pickhardt, M. et al. Curr. Alzheimer Res. 2, 219-226 (2005).

15. Noble, W. et al. Proc. Natl. Acad. Sci. USA 102, 6990-6995 (2005)

\title{
Imaging heterogeneity in synaptic transmission
}

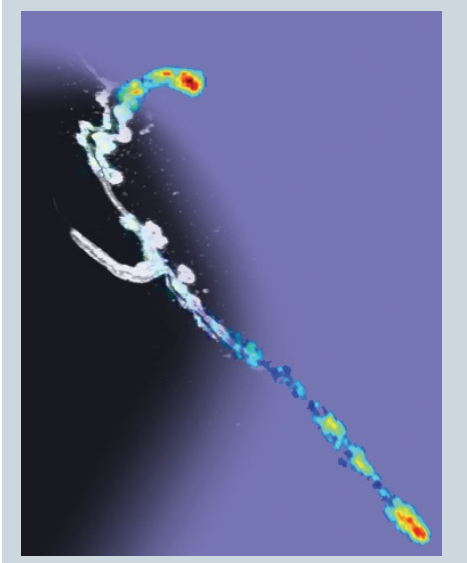

Neurons can form synapses with just one postsynaptic target or with hundreds, and an individual neuron can make single or multiple connections with each postsynaptic cell. To add to this diversity, connections also vary in strength. These multiple variables are difficult to measure electrophysiologically. On page 1188 of this issue, Guerrero et al. report a new optical approach to compare transmission from different connections of an individual neuron in the neuromuscular junction of the fruitfly Drosophila.

The authors created an innovative genetically encoded calcium reporter called SynapCam to selectively study calcium influx through glutamate receptors (which in Drosophila are permeable to $\mathrm{Ca}^{2+}$ ). They were able to target SynapCam postsynaptically, and image single boutons. They report that calcium signals through glutamate receptors were uniform within a given connection but varied considerably among different connections made by a single axon at the same neuromuscular junction. Moreover, they observed a gradient of transmission strength along axonal branches, with the strongest synapses at the terminal end of an axonal branch (picture).

Kalyani Narasimhan 